\title{
Autoconfianza y actitud hacia la enseñanza del emprendimiento
}

Impulsores clave de la intención emprendedora

Self-confidence and attitude towards entrepreneurship teaching Key driving factors of the entrepreneurial intention

Autoconfiança e atitude em relação ao ensino do empreendedorismo Principais motivadores da intenç̃o empreendedora

\section{DOI: https://doi.org/10.18861/cied.2021.12.2.3091}

\section{Sebastián Araya-Pizarro}

Universidad de La Serena

Chile

ORCID: 0000-0002-5857-8441

Recibido: 21/03/21

Aprobado: 11/05/2

Cómo citar: Araya-Pizarro, S. (2021). Autoconfianza y actitud hacia la enseñanza del emprendimiento: impulsores claves de la intención emprendedora. Cuadernos de Investigación Educativa, 12(2). https: //doi.org/10.18861/cied.2021.12.2.3091

\section{Resumen}

La enseñanza delemprendimiento es de vital importancia para el desarrollo socioeconómico de las naciones, especialmente por su incidencia en la intencion de emprender del alumnado. El presente estudio analiza los factores impulsores de la intencion emprendedora en estudiantes universitarios que cursan asignaturas de emprendimiento. A traves de un modelo de regresion logistica binaria aplicado a 85 estudiantes de una universidad publica de Chile se develo que la actitud favorable hacia la ensenanza del emprendimiento y la autoconfianza del discente inciden de manera positiva y significativa en la intencion de emprender. Tambien se determino que la carrera elegida y la experiencia laboral constituyen predictores relevantes. Se concluye que la intención emprendedora del estudiante aumenta cuando percibe que la enseñanza del emprendimiento es uth para su formacion como emprendedory cuando tiene el convencimiento intmo de que es capaz de emprender. Por lo demás, existe mayor probabilidad de que el estudiante emprenda si posee experiencia laboral cursa una carrera de perfil emprendedor. Se espera que los resultados aporten a una comprension mas profunda sobre los factores que llevan a los alumnos universitarios a emprender, evidenciándose asi la importancia de desarrollar programas de estudio que potencien la confianza del estudiantado y una actitud favorable hacia la enseñanza del emprendimiento

Palabras clave: intención emprendedora, enseñanza del emprendimiento, factores predictivos, estudiantes universitarios, regresión logistica.

\section{Abstract}

The teaching of entrepreneurship is of vital importance for the socio-economic development of nations, especially due to its impact on the students' entrepreneurial intention. This study analyzes the factors driving the interest in this subject in university students who take entrepreneurship courses. Through a binary logistic regression model applied to 85 students of entreprene state university, it was revealed that the favorable attitude towards the teaching on their entrepreneurial intention It was also determined that the chosen career and work experience are relevant predictors. It is concluded that the interest of students to undertake entrepreneurship increases when they perceive that the teaching of this topic is useful for their training as an entrepreneur, and when they have the deep conviction that they are capable of undertaking entrepreneurship. There is also a greater chance that the student will undertake entrepreneurship if they have work experience and are pursuing entrepreneurial studies. The results are expected to conthbute to a deeper understanding of the factors that lead university students to undertake chtrepreneurship, which evidences the importance of developing entrepreneurship.

Keywords: entrepreneurial intention, teaching entrepreneurship, predictive factors, university students, logistic regression

\section{Resumo}

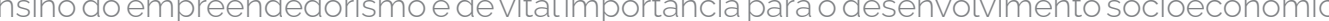
das naçoes, principalmente pelo seu impacto na intençao de empreender dos alunos. A presente pesquisa analisa os impulsionadores da intençao empreendedora em estudantes universitarios que fazem cursos de empreendedorismo. Mediante um padrăo de regressao ogistica binaria aplicado a 85 alunos de uma universidade publica chitena, foi possivet perceber que a atitude favoravel ao ensino do empreendedorismo e a autoconfiança do aluno têm um impacto positivo e significativo na intençao de empreender. Tambem fol determinado que o curso escolhido e a experiencia de trabatho sáo preditores relevantes. Conclui-se que a intençào enpreendedora do atuno se acrescenta quando percebe que o ensino do empreendedorismo que écapaz de que capaz de rîn se que os resultados contribuam para uma compreensão mais aprofundada dos fatores que evam os universitários a empreender evidenciando a importância do desenvelvimento de programas de estudos que acrescentem a confiança dos alunos e uma atitude favorável ao ensino do empreendedorismo

Palavras-chave: intenção empreendedora, ensino de empreendedorismo, fatores preditivos, Pstudantes universtitarios, regressão logistica 


\section{Introducción}

Históricamente los investigadores del emprendimiento han centrado sus esfuerzos en responder preguntas sobre quién es un emprendedor (enfoque de los rasgos), qué entorno lo forma (enfoque sociodemográfico) y por qué alguien elige convertirse en este tipo de persona (enfoque conductual). De este modo, las investigaciones primigenias sobre emprendimiento tuvieron como objetivo definir y caracterizar a los emprendedores y sus decisiones empresariales en función de los rasgos de personalidad (Brockhaus, 1982; McClelland, 1961). Actualmente se han registrado estudios sobre distintos atributos y habilidades específicos tales como la creatividad (Chia y Liang, 2016) o la inteligencia emocional (García-Cabrera et al., 2015; Mortan et al., 2014) que se unen a tradicionales variables predictoras de la intención emprendedora (IE) tales como la autoeficacia, las actitudes, los valores y las motivaciones (Fayolle et al., 2014; Echeverri-Sánchez et al., 2018; Law y Breznik, 2017; Wang et al., 2016).

Por lo demás, el estudio del perfil sociodemográfico se centra en las caracteristicas personales y del entorno social del individuo que influyen en su formación como emprendedor. Se supone que las personas con antecedentes similares también poseen características similares, y el razonamiento detrás de esto es bastante parecido al del enfoque de rasgos. Es decir, identificar las caracteristicas sociodemográficas de los emprendedores conocidos permitiría predecir la actividad empresarial en poblaciones desconocidas. Las variables demográficas que a menudo se examinan en el dominio del emprendimiento incluyen antecedentes personales y familiares tales como la edad, el género, la escolaridad, el nivel socioeconómico, la experiencia laboral y los modelos de referencia, entre otros (Liñán y Fayolle, 2015; Robinson et al., 1991; Veciana, 1999).

En el caso de la experiencia laboral, las investigaciones la señalan como un factor positivo para la creación yel desarrollo exitoso de los proyectos de emprendimiento. Se supone que el impacto sobre la intención emprendedora puede explicarse por una mejor detección de oportunidades empresariales, fruto del conocimiento del mercado y de las actividades productivas, alcanzado durante el ejercicio de la actividad profesional (Majid et al., 2011; Soriano y Castrogiovanni, 2012). Al respecto, Dencker et al. (2009) aclaran que no toda la experiencia tiene un efecto positivo y significativo en el deseo de emprender, dándose una correlación positiva solo en los casos en los que la experiencia ha sido en el mismo campo en el que se ha creado la empresa. Asimismo cabe destacar que la insatisfacción laboral, derivada de la falta de desafios u oportunidades promocionales, asi como la frustración y el aburrimiento a menudo operan como impulsores para la creación de una nueva empresa (Hisrich et al., 2017).

La educación en emprendimiento, por su parte, se ha convertido en un tema de interés universal, por lo que es posible encontrar diversas investigaciones que analizan el estado actual de su enseñanza (Čapienè y Ragauskaitè, 2017: Castillo, 1999; Gutiérrez et al., 2016; Herrera y Villalobos, 2018; Sánchez et al., 2017) y de su influencia sobre la intención emprendedora del estudiantado (Barba-Sánchez y Atienza-Sahuquillo, 2018; Díaz-García et al., 2015; Doğan, 2015; Hattab, 2014; Ismail et al., 2018; Mahendra et al., 2017; Maresch et al., 2016; Prasetio, 2016; Sondari, 2014; Soria-Barreto et al., 2016). En general existe una visión común de que la instrucción formal potencia la intención emprendedora de las personas (Karim, 2016). Por ello es posible advertir que cuanto más intensiva sea la enseñanza del emprendimiento en las instituciones de educación terciaria más probabilidades habrá de que el estudiantado haga el esfuerzo por empezar un nuevo negocio y, de esa manera, contribuir al desarrollo de la economía (Alvarez et al., 2006). Por ello se indica que la educación emprendedora tiene la ventaja de identificar y suscitar las vocaciones empresariales, potenciar el autoempleo y enlazar los sectores educativo y empresarial (Chapa, 2008). 
No obstante cabe señalar que la enseñanza del emprendimiento no se limita a la impartición de técnicas y procedimientos para la creación de empresas y su gestión (enfoque utilitarista), sino que persigue la formación integral de sujetos sociales capaces de emprender en todos los ámbitos de la actividad socioculturaly profesional. Así, la docencia universitaria tiene el desafío de garantizar el desarrollo cognitivo, actitudinal y valórico del alumnado, con base en el establecimiento de enfoques, estrategias pedagógicas y estilos de enseñanza que estimulen la participación activa del estudiante en la construcción de su propio aprendizaje (Aldana-Rivera et al., 2019).

En este contexto el presente estudio se planteó como objetivo analizar los factores que impulsan la intención emprendedora en estudiantes universitarios que cursan asignaturas de emprendimiento. En concreto se examina la incidencia de la autoconfianza del estudiante, la valoración de la enseñanza del emprendimiento y de un conjunto de rasgos personales como el sexo, la edad, el nivel de renta, la experiencia laboral y los modelos de referencia (familiares y emprendedores).

Cabe señalar que, en este estudio, la intención de emprender se configura en base a la noción de iniciativa empresarial (Liñán et al., 2011). De este modo establece una visión particular sobre cómo diferentes factores destacados por la literatura influyen en la posibilidad de que el alumnado emprenda y, por tanto, manifieste el ánimo de crear o dirigir una empresa propia.

Se espera que los resultados aporten a una comprensión más profunda sobre las variables que llevan a los estudiantes universitarios a emprender, contribuyendo a mejorar el diseño de los programas de emprendimiento que se desarrollan desde universidades y otras instituciones educativas.

\section{Metodología}

La investigación corresponde a un estudio de casos acotado a la medición de la intención emprendedora de los estudiantes de la materia Emprendimiento de una universidad pública chilena. El estudio utilizó un enfoque cuantitativo, de diseño no experimental, de tipo explicativo y de corte transversal.

La población estuvo conformada por el estudiantado de dos carreras del área de las ciencias administrativas (Ingeniería Comercial e Ingeniería en Administración de Empresas) que cursaban dos asignaturas semestrales obligatorias de emprendimiento (Actitud Emprendedora y Emprendimiento), con cupos promedio de 50 alumnos.

Se computó un tamaño muestral de 85 alumnos con base en una población total de 97 estudiantes, un margen de error de 3.8\%, un nivel de confianza del 95\% y una proporción del 50\% (heterogeneidad).

Los estudiantes fueron seleccionados a través de un muestreo probabilístico aleatorio simple. Como primer paso se tuvo acceso a la lista de estudiantes inscritos en los cursos de interés, los que fueron contactados para responder el cuestionario. A continuación, en la Tabla 1, figura la ficha técnica de la investigación. 
Tabla 1. Ficha técnica del proceso muestral estudio

\begin{tabular}{|l|l|}
\hline Detalle & Descripción \\
\hline Población & $\begin{array}{l}97 \text { estudiantes (55 alumnos del curso Actitud } \\
\text { Emprendedora de la carrera de Ingeniería Comercial y } \\
42 \text { estudiantes del curso Emprendimiento de la carrera } \\
\text { de Ingeniería en Administración de Empresas). }\end{array}$ \\
\hline Muestra & $\begin{array}{l}85 \text { alumnos (47 alumnos de Ingeniería Comercial y 38 } \\
\text { alumnos de Ingeniería en Administración de Empresas). }\end{array}$ \\
\hline Error muestral & $\pm 3.8 \%$ \\
\hline Nivel de confianza & $95 \%$ \\
\hline Muestreo & Aleatorio simple \\
\hline Recolección de datos & Encuesta presencial escrita y encuesta en línea \\
\hline
\end{tabular}

El cuestionario, validado a través del juicio de cinco expertos (profesionales con grado de doctor en Educación), se compuso de tres conjuntos de preguntas. El primer grupo se enfocó en caracterizar el perfil del alumnado (sexo, edad, situación laboral, nivel de ingresos, autoconfianza para emprender, conocimiento de emprendedores, padres o tutores empresarios). El segundo apartado examinó su percepción respecto de la educación emprendedora mediante una pregunta abierta ("Creo que la enseñanza del emprendimiento es") y tres preguntas de cerradas ("Utilidad para su vida futura", "Contribución al desarrollo de habilidades emprendedoras y potenciamiento de la creatividad" y "Aversión al riesgo"). Y la tercera sección, basada en el trabajo de Liñán et al. (2011), evaluó la intención emprendedora a través de tres cuestionamientos sobre su interés por iniciar una empresa, convertirse en emprendedor y dirigir su propio negocio. Cabe indicar que los dos últimos ítems utilizaron Escalas Likert de cinco puntos, que oscilaron desde muy en desacuerdo (1) a muy de acuerdo (5)

Los datos fueron analizados mediante el uso de una regresión logística binaria, un método estadístico que permite describir y estimar la relación entre una variable binaria dependiente (categórica) y las variables independientes del modelo (numéricas o categóricas), entregando un modo alternativo de expresar la probabilidad de ocurrencia de un evento de interés, denominado odds ratio (Cerda et al., 2013; Hair et al., 1999).

Para el modelo, la variable dependiente es la IE $(s i=1, n o=0)$ y las variables independientes se agruparon en categorías dicotómicas donde se consideraron los siguientes ítems: autoconfianza ( $(i=1 ; n o=0$ ), conocimiento de emprendedores ( $s i=1, n o=0)$, padres empresarios $(s i ́=1, n o=0)$ e importancia percibida sobre la

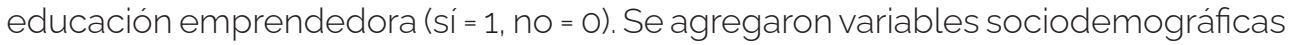
como el sexo (hombre $=1$, mujer $=0$ ), la edad (menor a $20=1,20$, o más =0), la situación laboral (si trabaja $=1$, no trabaja $=0$ ) y el nivel de ingresos (hasta CLP $1.500 .000=1$, más de CLP $1.500 .000=0$ ).

El modelo de regresión logística propuesto puede representarse bajo la siguiente estructura matemática:

$$
\log \left(\frac{p}{1-p}\right)=b_{0}+b_{1} x_{1}+b_{2} x_{2}+\cdots+b_{k} x_{k}
$$

donde p representa la probabilidad de que el estudiante posea la intención de emprender (situación de interés). Según el valor de las variables explicativas puede estimarse directamente la probabilidad de que ocurra el evento de interés del modo que se presenta a continuación: 


$$
\hat{p}=\left(\frac{e^{\hat{b}_{0}+\hat{b}_{1} x_{1}+\hat{b}_{2} x_{2}+\cdots+\hat{b}_{k} x_{k}}}{1-e^{\hat{b}_{0}+\hat{b}_{1} x_{1}+\hat{b}_{2} x_{2}+\cdots+\hat{b}_{k} x_{k}}}\right)
$$

El poder predictivo y ajuste del modelo propuesto fue medido con la prueba omnibus sobre los coeficientes del modelo, la prueba de Hosmeer \& Lemeshow, el seudocuadrado R de McFadden y el R cuadrado de Nagelkerke.

Asimismo, el examen de la pregunta abierta incluyó el análisis de contenido (categórico descriptivo) a través de la codificación y categorización de las respuestas. Las categorias fueron construidas desde una perspectiva inductiva sin contemplar categorías analíticas predefinidas teóricamente. Inicialmente se realizó una definición de categorias centrales halladas a partir de la reducción sistemática de las unidades temáticas que surgieron de la interpretación de los propios relatos del alumnado. Más tarde estas fueron sometidas a un análisis estadistico de datos textuales (frecuencia de palabras) y a la construcción de un mapa mental de los principales tópicos extraidos.

Por último cabe destacar que los datos cuantitativos y cualitativos del estudio fueron procesados a través de los programas IBM SPSS Statistics y ATLAS.ti, respectivamente.

\section{Resultados}

\section{Caracterización de la muestra}

Los participantes del estudio fueron 85 alumnos de las carreras de Ingeniería Comercial (55\%) e Ingeniería en Administración de Empresas (45\%) que estudiaban en una universidad pública chilena. La caracterización de la muestra se presenta en la Tabla 2, en la que se aprecia que el alumnado se vincula con el emprendimiento principalmente a través de las experiencias de personas que han emprendido fuera de sus padres o tutores (solo el 26\% tiene padres empresarios).

Tabla 2. Caracterización de la muestra $(n=85)$

\begin{tabular}{|c|c|c|c|}
\hline Variable & Segmento & Total & Porcentaje \\
\hline \multirow[t]{2}{*}{ Género } & Masculino & 24 & 28.2 \\
\hline & Femenino & 61 & 71.8 \\
\hline \multirow[t]{3}{*}{ Edad } & Menos de 21 años & 37 & 43.5 \\
\hline & 21 años & 25 & 29.4 \\
\hline & Más de 21 años & 23 & 27.1 \\
\hline Renta mensual hogar & Menos de 350.000 & 15 & 17.6 \\
\hline \multirow[t]{5}{*}{ (en CLP) } & Entre 350.000 y 700.000 & 31 & 36.5 \\
\hline & Entre 700.001 y 1.050 .000 & 20 & 23.5 \\
\hline & Entre 1.050 .001 y 1.400 .000 & 2 & 2.4 \\
\hline & Entre 1.400 .001 y 1.750 .000 & 7 & 8.2 \\
\hline & Más de 1.750 .000 & 10 & 11.8 \\
\hline \multirow[t]{2}{*}{ Experiencia laboral } & Sí & 68 & 80.0 \\
\hline & No & 17 & 20.0 \\
\hline \multirow[t]{2}{*}{ Padres empresarios } & Sí & 22 & 25.9 \\
\hline & No & 63 & 74.1 \\
\hline \multirow[t]{2}{*}{ Conoce emprendedores } & Sí & 73 & 85.9 \\
\hline & No & 12 & 14.1 \\
\hline
\end{tabular}




\section{Intención emprendedora}

Los hallazgos del escrutinio de la intención de emprender de los estudiantes mostraron que la mayoría han pensado en iniciar una empresa (79\%) y que están dispuestos a esforzarse por crear y dirigir su propio negocio (73\%). Asimismo, más de la mitad del alumnado reconoció tener como objetivo profesional convertirse en un emprendedor (52\%). Ver la Figura 1.

Figura 1. Intención de emprender del estudiantado

\begin{tabular}{|c|c|c|}
\hline $\begin{array}{c}\text { Pienso seriamente en } \\
\text { iniciar una empresa }\end{array}$ & $\begin{array}{l}\text { Quiero convertirme en un } \\
\text { emprendedor }\end{array}$ & $\begin{array}{l}\text { Deseo crear y dirigir mi } \\
\text { propia empresa }\end{array}$ \\
\hline & $\begin{array}{l}\text { No } \\
48 \%\end{array}$ & 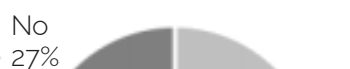 \\
\hline
\end{tabular}

Asimismo, el examen descriptivo de la intención emprendedora según las variables de caracterización del alumnado (Tabla 3) reveló lo siguiente:

- Los estudiantes de la carrera de ingeniería en administración poseen mayor IE que los estudiantes de ingenieria comercial (86\% versus 73\%). Asimismo muestran mayores deseos de convertirse en emprendedores (68\% versus $40 \%$ ) y de crear sus propias empresas ( $84 \%$ versus $65 \%$ ).

- Los estudiantes de más edad (mayores de 21 años) tienen más intenciones de emprender que los estudiantes más jóvenes (91\% versus 74\% promedio). Muestran mayores deseos de desarrollarse como emprendedores $165 \%$ versus $48 \%$ promedio) y de crear sus propias empresas ( $87 \%$ versus $67 \%$ ).

- Los estudiantes de menores ingresos (inferior a CLP 700 001), a diferencia de aquellos con más renta, poseen mayor IE (84\% versus 72\%), más deseos de convertirse en emprendedores (61\% versus $41 \%$ ) y mayor interés en crear sus propias empresas (76\% versus $72 \%$ ).

- Los estudiantes que poseen experiencia laboral, en contraposición con aquellos sin experiencia laboral, tienen mayores intenciones de emprender ( $81 \%$ versus $71 \%$ ), más deseos de ser emprendedores (59\% versus $24 \%$ ) y de crear sus propias empresas ( $79 \%$ versus $47 \%$ ).

- Los estudiantes sin padres empresarios muestran mayor IE que aquellos que provienen de una familia de empresarios (81\% versus $73 \%$ ). También muestran mayor deseo de convertirse en emprendedores (54\% versus 45\%) y de crear su propia empresa ( $76 \%$ versus $64 \%$ ).

- Los estudiantes que conocen emprendedores poseen niveles más altos de IE (84\% versus 50\%), muestran mayor deseo de seguir una carrera de emprendedor (53\% versus $42 \%$ ) y de iniciar y dirigir sus propias empresas (78\% versus $42 \%$ ).

- En cuanto al género se observó que, a diferencia de los hombres, en general las mujeres piensan menos en emprender ( $7 \%$ versus 0\%), muestran menos deseos de ser emprendedoras (13\% versus $8 \%$ ) y menos ánimo de crear su propia empresa ( $8 \%$ versus $4 \%$ ). Esto revela que los estudiantes que tienen bajos niveles de IE y, por tanto, un mayor rechazo por emprender, son de género femenino. 
Tabla 3. Intención emprendedora del estudiantado según variables de caracterización (\%)

\begin{tabular}{|c|c|c|c|c|c|c|c|c|c|}
\hline \multirow[b]{2}{*}{ Preguntas } & \multicolumn{3}{|c|}{ Pienso emprender } & \multicolumn{3}{|c|}{$\begin{array}{l}\text { Quiero ser } \\
\text { emprendedor }\end{array}$} & \multicolumn{3}{|c|}{$\begin{array}{l}\text { Deseo crear mi } \\
\text { empresa }\end{array}$} \\
\hline & Baja & Media & Alta & Baja & Media & Alta & Baja & Media & Alta \\
\hline Total & 5 & 16 & 79 & 12 & 36 & 52 & 7 & 20 & 73 \\
\hline \multicolumn{10}{|l|}{ Carrera } \\
\hline Ing. en Administración & 5 & 8 & 86 & 11 & 22 & 68 & 11 & 5 & 84 \\
\hline Ingeniería Comercial & 4 & 23 & 73 & 13 & 48 & 40 & 4 & 31 & 65 \\
\hline \multicolumn{10}{|l|}{ Género } \\
\hline Masculino & 0 & 21 & 79 & 8 & 38 & 54 & 4 & 21 & 75 \\
\hline Femenino & 7 & 15 & 79 & 13 & 36 & 51 & 8 & 20 & 72 \\
\hline \multicolumn{10}{|l|}{ Edad } \\
\hline Menos de 21 años & 3 & 22 & 76 & 14 & 43 & 43 & 11 & 19 & 70 \\
\hline 21 años & 12 & 16 & 72 & 16 & 32 & 52 & 8 & 28 & 64 \\
\hline Más de 21 años & 0 & 9 & 91 & 4 & 30 & 65 & 0 & 13 & 87 \\
\hline \multicolumn{10}{|l|}{ Ingreso mensual del hogar } \\
\hline Menos de CLP 350.000 & 7 & 13 & 80 & 7 & 33 & 60 & 7 & 13 & 80 \\
\hline CLP $350.000-700.000$ & 6 & 6 & 87 & 10 & 29 & 61 & 6 & 23 & 71 \\
\hline CLP $700.001-1.050 .000$ & 0 & 25 & 75 & 10 & 55 & 35 & 0 & 30 & 70 \\
\hline Más de CLP 1.050.000 & 5 & 26 & 68 & 21 & 32 & 47 & 16 & 11 & 74 \\
\hline \multicolumn{10}{|l|}{ Experiencia laboral } \\
\hline Sí & 4 & 15 & 81 & 12 & 29 & 59 & 6 & 15 & 79 \\
\hline No & 6 & 24 & 71 & 12 & 65 & 24 & 12 & 41 & 47 \\
\hline \multicolumn{10}{|l|}{ Padres empresarios } \\
\hline Si & 0 & 27 & 73 & 14 & 41 & 45 & 9 & 27 & 64 \\
\hline No & 6 & 13 & 81 & 11 & 35 & 54 & 6 & 17 & 76 \\
\hline \multicolumn{10}{|l|}{ Conoce emprendedores } \\
\hline Sí & 3 & 14 & 84 & 10 & 37 & 53 & 5 & 16 & 78 \\
\hline No & 17 & 33 & 50 & 25 & 33 & 42 & 17 & 42 & 42 \\
\hline
\end{tabular}

No obstante cabe destacar que las pruebas de medias ejecutadas (ANOVA de un factor) solo mostraron diferencias significativas entre el nivel de IE de los estudiantes con el conocimiento de emprendedores, $F(1,83)=7.21, p<.01$, y una significancia débil con la experiencia laboral, $F(1,83)=2.96, p=.089$. Por tanto, primeramente se evidencia que los modelos de referencia desempeñan un papel importante en el fortalecimiento de la intención de emprender de los alumnos (Gómez-Araujo et al., 2015; Zhu et al., 2019). Así, en línea con lo expuesto por la Teoría del Aprendizaje Social de Bandura (1977) estos referentes actuarian dirigiendo la atención del individuo hacia el emprendimiento, en un aporte conjunto con los conocimientos y habilidades necesarios para crear una nueva empresa, partiendo del apoyo hacia el surgimiento de comportamientos emprendedores. En segundo término se observa que la experiencia laboral influye positivamente en la IE tal como ha sido propuesto por otros autores (Majid et al., 2011; Soriano y Castrogiovanni, 2012). 


\section{Valoración de la enseñanza del emprendimiento}

La valoración de la enseñanza del emprendimiento se evaluó mediante tres preguntas que analizaron la utilidad percibida de estudiar emprendimiento ("creo que aprender sobre emprendimiento es útil para mi vida futura"), el aporte de esta enseñanza al desarrollo de habilidades emprendedoras ("creo que las habilidades emprendedoras se adquieren a través de la formación") y su contribución para mejorar la creatividad y aversión al riesgo ("creo que los cursos de emprendimiento mejoran la capacidad para generar ideas creativas y enfrentar el riesgo").

Por un lado, los resultados expuestos en la Figura 2 revelan que la mayoría de los estudiantes creen que la enseñanza del emprendimiento es útil para su vida futura (97\%), que los cursos de emprendimiento mejoran la capacidad para generar ideas creativas y enfrentar riesgos (97\%), y que las habilidades emprendedoras se adquieren a través de la formación (77\%). Respecto de este último tópico cabe destacar que el 11\% de los educandos considera que la contribución de un curso en emprendimiento es exigua.

Figura 2. Valoración de la enseñanza del emprendimiento (\%)

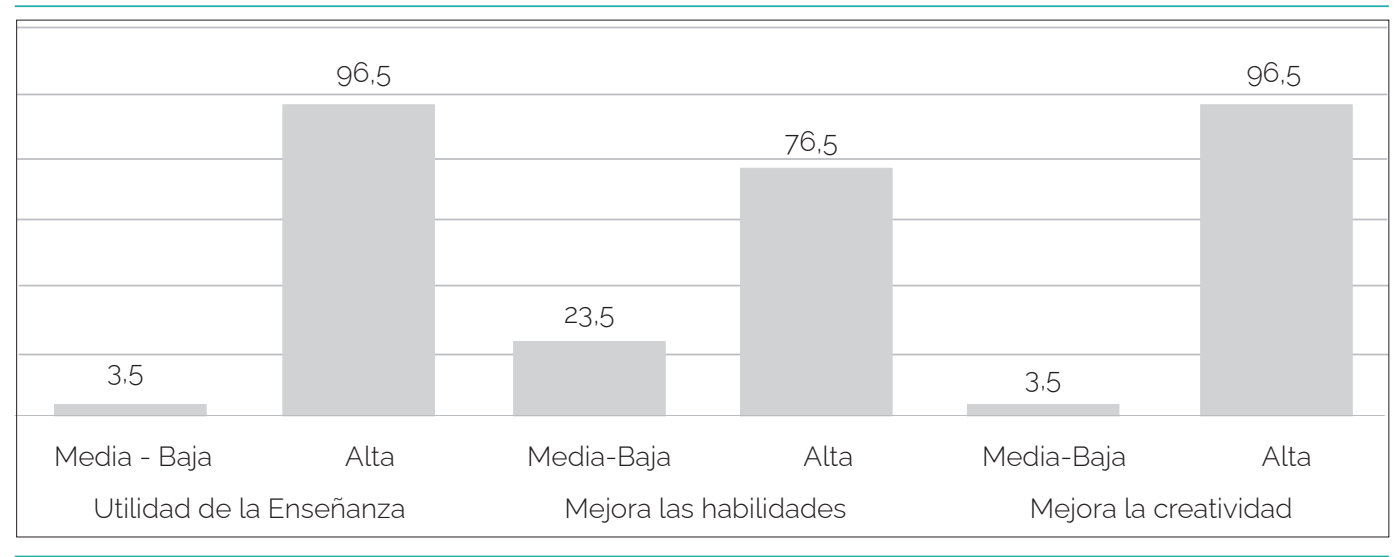

Por otro lado, el análisis de la valoración de la enseñanza del emprendimiento por variables de caracterización, presentado en la Tabla 4, mostró lo siguiente:

- Los estudiantes, en general, perciben que la enseñanza del emprendimiento será útil para su vida, con altos niveles de apreciación que oscilan entre el 92\% y $100 \%$.

- Los estudiantes que consideran que la enseñanza del emprendimiento contribuye al desarrollo de las habilidades emprendedoras pertenecen a la carrera de Ingeniería Comercial (84\%), son del sexo femenino (79\%), de 21 años de edad (92\%), poseen ingresos menores a CLP 700000 (79\% promedio) y conocen a emprendedores (78\%). Asimismo, más allá de que tengan experiencia laboral o padres empresarios, los alumnos valoran su aporte de forma similar (76\%).

- Por lo demás, independientemente de sus características, los estudiantes, perciben que la enseñanza del emprendimiento ayuda a mejorar la capacidad para generar ideas creativas y enfrentar el riesgo, con apreciaciones que fluctúan entre el 92\% y el 100\%.

El contraste de medias entre variables (prueba t para muestras independientes) solo detectó diferencias significativas en la valoración percibida sobre la utilidad de la enseñanza según su conocimiento de personas emprendedoras, t(83) = $-2.13, p=.036$. Ello confirma que los modelos de referencia desempeñan un papel importante, no solo en el fortalecimiento de la IE sino también en su apreciación sobre el beneficio de la enseñanza del emprendimiento para su desarrollo personal. 
Tabla 4. Valoración de la enseñanza del emprendimiento (\%)

\begin{tabular}{|c|c|c|c|c|c|c|c|c|c|}
\hline \multirow[b]{2}{*}{ Preguntas } & \multicolumn{3}{|c|}{$\begin{array}{c}\text { Utilidad de la } \\
\text { enseñanza }\end{array}$} & \multicolumn{3}{|c|}{ Mejora habilidades } & \multicolumn{3}{|c|}{ Mejora creatividad } \\
\hline & Baja & Media & Alta & Baja & Media & Alta & Baja & Media & Alta \\
\hline Total & 2 & 1 & 96 & 11 & 13 & 76 & 2 & 1 & 96 \\
\hline \multicolumn{10}{|l|}{ Carrera } \\
\hline Ing. en Administración & 5 & 0 & 95 & 11 & 5 & 84 & 5 & 0 & 95 \\
\hline Ingenieria Comercial & 0 & 2 & 98 & 10 & 19 & 71 & 0 & 2 & 98 \\
\hline \multicolumn{10}{|l|}{ Género } \\
\hline Masculino & 0 & 4 & 96 & 8 & 21 & 71 & 0 & 0 & 100 \\
\hline Femenino & 3 & 0 & 97 & 11 & 10 & 79 & 3 & 2 & 95 \\
\hline \multicolumn{10}{|l|}{ Edad } \\
\hline Menos de 21 años & 3 & 0 & 97 & 14 & 16 & 70 & 3 & 3 & 95 \\
\hline 21 años & 4 & 4 & 92 & 4 & 4 & 92 & 4 & 0 & 96 \\
\hline Más de 21 años & 0 & 0 & 100 & 13 & 17 & 70 & 0 & 0 & 100 \\
\hline \multicolumn{10}{|l|}{ Ingreso mensual del hogar } \\
\hline Menos de CLP 350.000 & 7 & 0 & 93 & 7 & 20 & 73 & 7 & 0 & 93 \\
\hline CLP 350.000-700.000 & 3 & 0 & 97 & 13 & 3 & 84 & 3 & 0 & 97 \\
\hline CLP 700.001-1.050.000 & 0 & 5 & 95 & 15 & 15 & 70 & 0 & 0 & 100 \\
\hline Más de CLP 1.050.000 & 0 & 0 & 100 & 5 & 21 & 74 & 0 & 5 & 95 \\
\hline \multicolumn{10}{|l|}{ Experiencia laboral } \\
\hline Sí & 3 & 1 & 96 & 10 & 13 & 76 & 3 & 1 & 96 \\
\hline No & 0 & 0 & 100 & 12 & 12 & 76 & 0 & 0 & 100 \\
\hline \multicolumn{10}{|l|}{ Padres empresarios } \\
\hline Si & 0 & 5 & 95 & 14 & 9 & 77 & 0 & 0 & 100 \\
\hline No & 3 & 0 & 97 & 10 & 14 & 76 & 3 & 2 & 95 \\
\hline \multicolumn{10}{|l|}{ Conoce emprendedores } \\
\hline Si & 1 & 1 & 97 & 10 & 12 & 78 & 1 & 1 & 97 \\
\hline No & 8 & 0 & 92 & 17 & 17 & 67 & 8 & 0 & 92 \\
\hline
\end{tabular}

Cabe indicar que los estudiantes también fueron consultados respecto de la finalidad de la enseñanza del emprendimiento. Sus respuestas evidenciaron que su concepción se asocia a la entrega de conocimientos para emprender y lograr la independencia económica, con un fuerte componente técnico que incluye la preparación en métodos y herramientas para la creación de empresas, concreción de ideas de negocios y toma de decisiones. Asimismo los alumnos consideran que permite desarrollar habilidades emprendedoras que contribuyen a identificar oportunidades, generar nuevas ideas y solucionar problemas reales de la sociedad. Por lo demás se percibe como una alternativa para surgir, que posibilita desarrollarse personal y profesionalmente para progresar en la vida. Asimismo se atribuye a la enseñanza del emprendimiento el potenciar una actitud favorable hacia la tarea de emprender, lo que constituye una fuente de motivación y medio para cambiar la mentalidad de sus participantes. Véase la Figura 3. 
Figura 3. Mapa mental sobre la percepción de la enseñanza del emprendimiento

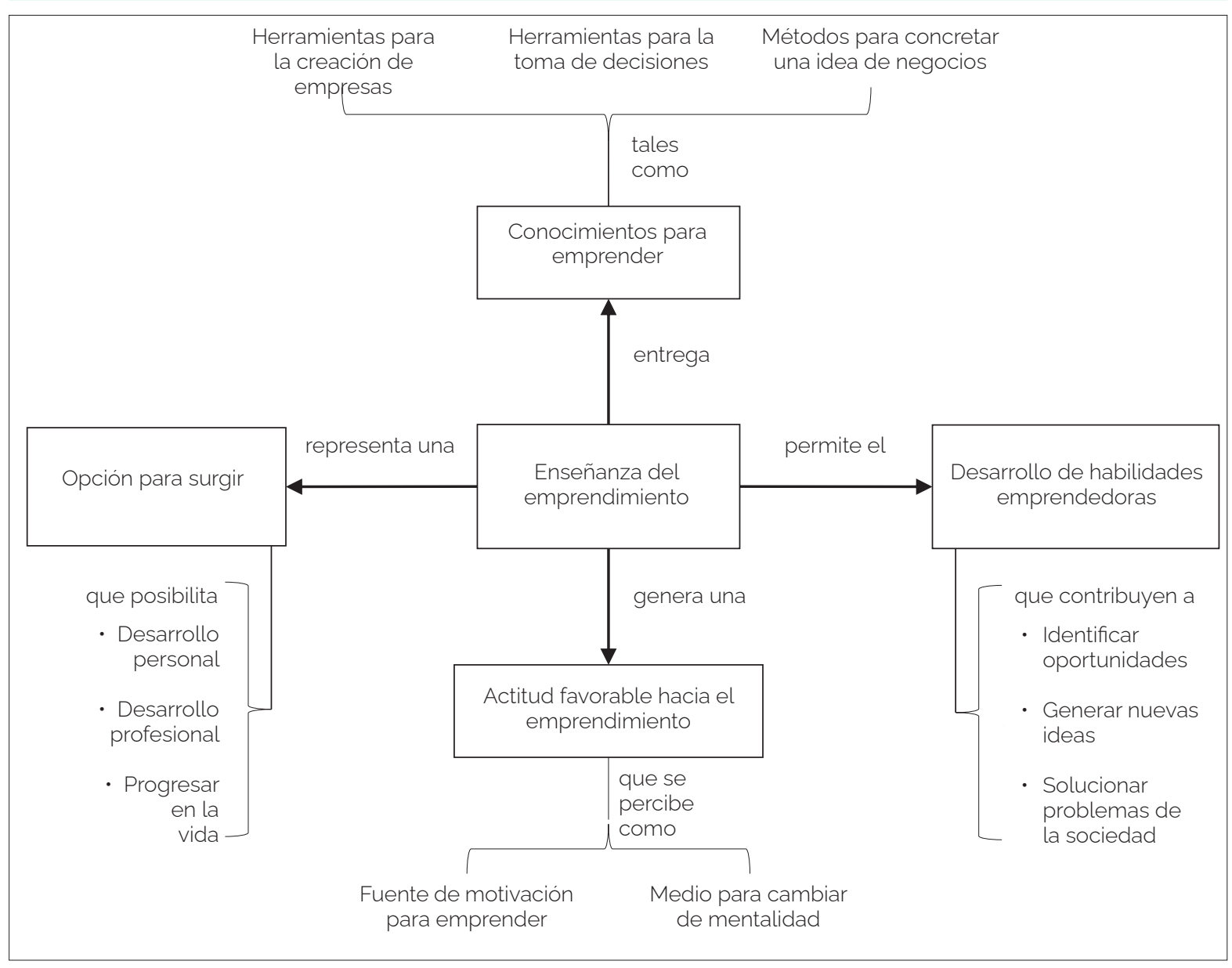

Finalmente, la Tabla 5 recoge algunos de los testimonios del estudiantado agrupados de acuerdo con los cuatro conceptos clave resultantes del análisis de contenido. 


\begin{tabular}{|c|c|}
\hline Dimensión & Testimonio \\
\hline $\begin{array}{l}\text { Conocimientos para } \\
\text { emprender }\end{array}$ & $\begin{array}{l}\text { - Tener más herramientas para desarrollar una empresa } \\
\text { - Facilitar métodos para comenzar un negocio } \\
\text { - Tener el conocimiento y herramientas para generar un negocio } \\
\text { - Tener las herramientas para crear una empresa en el futuro } \\
\text { - Conocer la forma de crear tu propio negocio } \\
\text { - Tener los conocimientos necesarios para la creación de una empresa }\end{array}$ \\
\hline $\begin{array}{l}\text { Desarrollo de } \\
\text { habilidades } \\
\text { emprendedoras }\end{array}$ & $\begin{array}{l}\text { - Ampliar las capacidades de poder emprender } \\
\text { - Innovar, crear, adaptar cambios y conocer habilidades } \\
\text { - Desarrollar un sinfín de habilidades } \\
\text { - La capacidad para generar ideas que ayuden a resolver diferentes } \\
\text { problemas }\end{array}$ \\
\hline $\begin{array}{l}\text { Actitud favorable } \\
\text { hacia el } \\
\text { emprendimiento }\end{array}$ & $\begin{array}{l}\text { - Motivarnos a emprender } \\
\text { - Cambiar mi visión acerca de este ámbito motivándome a llegar a } \\
\text { - Abrir la mente de los estudiantes a nuevos horizontes y metas } \\
\text { - Abrir tu mente, salir de la caja }\end{array}$ \\
\hline Opción para surgir & $\begin{array}{l}\text { - Crecer en la vida } \\
\text { - Crecer en el ámbito laboral } \\
\text { - Progresar de manera significativa } \\
\text { - Abre puertas para trabajar } \\
\text { - Surgir cuando crees que se te cerraron todas las puertas }\end{array}$ \\
\hline
\end{tabular}

\section{Factores predictivos de la intención emprendedora}

Los resultados del modelo de regresión logística binaria mostraron que los factores que afectan de manera significativa la intención emprendedora son la autoconfianza ( $p<.001$ ) y la valoración de la educación emprendedora $(p<$ .05). Asimismo, en cuanto a los rasgos del estudiante resultaron significativas la carrera elegida $(p<.05)$ y la experiencia laboral $(p<.10)$. En concreto se halló que la intención emprendedora del alumnado aumenta de la siguiente forma: 12 veces si posee autoconfianza $(O R=12.77)$, 4 veces si percibe que la enseñanza del emprendimiento es valiosa $(\mathrm{OR}=3.62), 6$ veces si cuenta con experiencia laboral $(\mathrm{OR}=6.16)$ o si estudia ingeniería en administración $(\mathrm{OR}=6.31)$.

Cabe indicar que el poder explicativo del modelo predictivo fue del $48 \%$ de la variabilidad de la IE (R2 de Nagelkerke $=.48$ ). En total, el modelo estima un porcentaje correcto del $75 \%$ de los casos, con una capacidad de discriminación adecuada ( $A \cup C=.85$ ). Asimismo, la prueba de Hosmer-Lemeshow no resultó ser significativa, lo que confirma que no existe evidencia para considerar que los resultados estimados difieran de los observados $\left(x^{2}=7.55, p=37\right.$. Véase la Tabla 6. 
Tabla 6. Regresión logística binaria con las variables del modelo

\begin{tabular}{|c|c|c|c|c|c|}
\hline Variable & $\beta$ & E.T. & Wald & Sig. & $\operatorname{Exp}(\beta)$ \\
\hline Carrera (Ing. Administración) & 1.842 & 0.761 & 5.858 & $.016^{* *}$ & 6.307 \\
\hline Género (masculino) & 1,257 & 0.769 & 2.670 & .102 & 3.514 \\
\hline Edad (<21 años) & 0.801 & 0.568 & 1.990 & .158 & 2.227 \\
\hline Nivel de renta (< CLP 700.000) & 0.166 & 0.622 & 0.071 & .789 & 1.181 \\
\hline Experiencia laboral (Si) & 1.819 & 0.866 & 4.413 & $.036^{* *}$ & 6.163 \\
\hline Autoconfianza (Si) & 2.547 & 0.742 & 11.771 & $.001^{* * *}$ & 12.774 \\
\hline Padres empresarios (Si) & -0.101 & 0.643 & 0.025 & .875 & 0.904 \\
\hline Conoce emprendedores (Si) & -0.819 & 0.878 & 0.871 & .351 & 0.441 \\
\hline Valora la educación emprendedora (Sí) & 1.286 & 0.565 & 5.190 & $.023^{* *}$ & 3.619 \\
\hline Constante & -4.690 & 1.433 & 10.718 & $.001^{* * *}$ & 0.009 \\
\hline Prueba de Hosmer y Lemeshow & $\chi^{2}=7.553$ & Sig. $=.374$ & & & \\
\hline $\mathrm{R}^{2}$ de Cox y Snell & .357 & & & & \\
\hline $\mathrm{R}^{2}$ de Nagelkerke & .476 & & & & \\
\hline \multirow[t]{2}{*}{ Curva COR } & Área & E.T. & Sig. & Lim. inf. & Lim. sup. \\
\hline & .848 & 0.041 & $.000^{* * *}$ & .768 & .928 \\
\hline
\end{tabular}

* Significativo al 10\%, ${ }^{* *}$ Significativo al $5 \%,{ }^{* * *}$ Significativo al $1 \%$

Estos hallazgos muestran que la intención emprendedora del estudiante aumenta cuando percibe que la enseñanza del emprendimiento es útil para su formación como emprendedor y cuando tiene el convencimiento íntimo de que es capaz de emprender. Por lo demás, existe mayor probabilidad de emprender si el estudiante posee experiencia laboral y cursa una carrera de perfil emprendedor.

De lo anterior es posible confirmar que la actitud favorable hacia la enseñanza del emprendimiento es fundamental para aumentar la IE de los estudiantes y un medio importante para potenciar su autoconfianza. Tal como ha sido expuesto por diversos autores (Arenius y Minniti, 2005; Busenitz et al., 2000; Gómez-Araujo et al., 2015; Hogarth y Karelaia, 2012; Krueger y Brazeal, 1994) la autoconfianza en los conocimientos y habilidades emprendedores llega a ser un factor psicológico crucial para la creación de nuevas empresas (McGee et al., 2009; Van Praag y Cramer, 2001).

De este modo se aprecia que, como variable efectiva esencial de la motivación, la actitud desempeña un papel preponderante en el proceso de enseñanzaaprendizaje delemprendimiento ya que, como se evidencia en la literatura, una mala predisposición puede empeorar hasta el punto de que descienda el rendimiento académico de los discentes o de que estos abandonen por completo el estudio de una asignatura (Araya-Pizarro y Avilés-Pizarro, 2020; Minera, 2009). De allí la relevancia de considerar una formación orientada a impartir conocimiento sobre emprendimiento e influenciar las actitudes hacia intenciones emprendedoras, es decir, brindar educación para concientizar sobre el emprendimiento (Liñán, 2004).

Asimismo es posible señalar a la experiencia laboral como un factor favorable para la creación y el desarrollo exitoso de los proyectos de emprendimiento. 
Este impacto positivo sobre la intención emprendedora podría explicarse, por una parte, por la mayor capacidad para detectar oportunidades empresariales, fruto del conocimiento del mercado y del sector, adquirido en el ejercicio de la actividad profesional (Majid et al., 2011; Soriano y Castrogiovanni, 2012); o por otra, por la insatisfacción laboral derivada de la falta de desafíos u oportunidades promocionales experimentada en su vida profesional, que a menudo opera como impulsora para la creación de una nueva empresa (Hisrich et al., 2017).

\section{Conclusiones}

De los hallazgos del estudio sobre los factores que impulsan la intención emprendedora de los estudiantes se concluye que el deseo de emprender es más fuerte entre los discentes que poseen el convencimiento intimo de que son capaces de emprender (autoconfianza), perciben que la educación emprendedora es útil para su desarrollo personal-profesional, y cuentan con la experiencia laboral que los lleva a valorar la independencia y la autonomía para la toma de decisiones.

Asimismo se confirma que la enseñanza del emprendimiento se considera importante no solo para la obtención de conocimientos sobre emprendimiento sino también para el desarrollo de competencias emprendedoras, es decir, de aquellas capacidades, destrezas, habilidades y aptitudes que hacen posible que quien emprende actúe para materializar una idea de negocios de forma rentable. De este modo existe una visión común en cuanto a que, para desarrollar un espíritu emprendedor, los conocimientos teóricos sobre la materia deben ser complementados con una formación en habilidades o competencias características del emprendedor.

En este sentido, con la finalidad de mejorar la actitud del discente hacia la enseñanza del emprendimiento se plantea que esta debe ser motivadora y, por tanto, debe aplicarse una metodología que inspire a emprender. Ello implica abordar los aspectos positivos y negativos del emprendimiento en la proporción necesaria para que el estudiantado perciba que emprender es factible y que las restricciones existentes son superables. Por tales motivos se recomienda establecer metodologías activas, altamente participativas, que permitan a los estudiantes desarrollar las competencias técnicas requeridas para la generación de modelos de negocios sustentables y la creación de empresas, así como desarrollar competencias consideradas clave para emprender tales como la creatividad, las relaciones sociales, el liderazgo, las redes y el trabajo en equipo. También se torna positiva la realización de charlas y visitas a emprendedores; actividades con alta aceptación entre estudiantes y que resultaron medios importantes de acercamiento al mundo del emprendimiento.

En cuanto a las limitaciones de la investigación cabe indicar, en primer lugar, que se utilizó una muestra pequeña que evidencia la necesidad de contar con un tamaño de mayor representación del universo de estudio. Y, en segundo lugar, que el instrumento de recolección de datos utilizado (cuestionario) está basado en percepciones de los estudiantes, por lo que obedecen a un componente subjetivo que está sujeto a sesgos resultantes del método de medición usado (varianza común del método), y a la forma en la que el educando plantea sus respuestas. 
Finalmente, de los resultados del estudio se desprenden líneas de investigación futuras tales como:

1) Analizar, desde una perspectiva cuantitativa, la incidencia de otras variables que pueden actuar como mediadoras de la intención emprendedora, como las metodologías de enseñanza, el estilo de aprendizaje, el conocimiento disciplinar específico y la duración del curso de educación en emprendimiento.

2) Desarrollar un estudio de enfoque cualitativo o mixto que permita conocer en profundidad cuáles son aquellos aspectos que influyen en mayor medida en la potenciación de la actitud del estudiante, así como analizar la efectividad de ciertas metodologías y prácticas pedagógicas en el aprendizaje del estudiantado (ABP, método de casos, gamificación, etc.).

3) Replicar este estudio utilizando otras muestras de mayor tamaño y de otros contextos, regiones o paises para determinar si las diferencias culturales son factores que afectan la intención empresarial.

4) Podrian desarrollarse estudios longitudinales que permitieran medir la evolución de los factores predictivos de la intención emprendedora.

Nota:

Aprobación final del artículo: editora responsable Mag. Verónica Zorrilla de San Martín.

Contribución de autoria: El artículo es 100 \% producción del autor (concepción del trabajo, diseño de la investigación, recolección de datos, procesamiento, análisis, elaboración y corrección del documento). 


\section{Referencias bibliográficas}

ALDANA-RIVERA, E., TAFUR-CASTILLO, J., GIL, I. y MEJíA, C. (2019). Práctica pedagógica de emprendimiento en docentes de educación superior en Institución Educativa Universitaria de Barranquia (sic). Revista AVFT, 38(2), 9-18. http://www.revistaavft.com/images/revistas/2019/avft_2_2019/2practica_ pedagogica_emprendimiento.pdf

ALVAREZ, R. D., DENOBLE, A. F., \& JUNG, D. (2006). Educational Curricula and Self-Efficacy: Entrepreneurial Orientation and New Venture Intentions among University Students in Mexico ICurrículo educativo y autoeficacia: orientación emprendedora e intenciones de nuevos emprendimientos entre estudiantes universitarios en México]. En: Galbraith, C.S. and Stiles, C.H. (Ed.) Developmental Entrepreneurship: Adversity, Risk, and Isolation (International Research in the Business Disciplines, Vol. 5), Emerald Group Publishing Limited, 379-403. https:// doi.org/10.1016/S1074-7877(06)05019-7

ARAYA-PIZARRO, S. C. \& AVILÉS-PIZARRO, N. B. (2020). Rendimiento académico en estudiantes de ciencias empresariales: ¿cómo influyen los factores actitudinales, pedagógicos y demográficos? Zona Próxima, 33, 70-97. https:// doi.org/10.14482/zp.33.658

ARENIUS, P., \& MINNITI, M. (2005). Perceptual Variables and Nascent Entrepreneurship [Variables perceptuales y emprendimiento naciente]. Small Business Economics, 24(3), 233-247. https://doi.org/10.1007/s11187-005-1984-X

BANDURA, A. (1977). Self-efficacy: Toward a Unifying Theory of Behavioral Change [Autoeficacia: hacia una teoría unificadora del cambio de comportamiento]. American Psychological Association, 84(2), 191-215. https://doi.org/10.1037/0033295X.84.2.191

BARBA-SÁNCHEZ, V., \& ATIENZA-SAHUQUILLO, C. (2018). Entrepreneurial Intention among Engineering Students: The role of Entrepreneurship Education IIntención emprendedora entre estudiantes de ingeniería: el rol de la educación emprendedoral. European Research on Management and Busines Economics, 24(1), 53-61. https://doi.org/10.1016/j.iedeen.2017.04.001

BROCKHAUS, R. (1982). The Psychology of the Entrepreneur ILa psicologia del emprendedor]. Encyclopedia of entrepreneurship, 39-57.

BUSENITZ, L. W., GÓMEZ, C., \& SPENCER, J. W. (2000). Country Institutional Profiles: Unlocking Entrepreneurial Phenomena IPerfiles institucionales de los paises: desbloqueando los fenómenos emprendedores]. Academy of Management Journal, 43(5), 994-1003. https://www.jstor.org/stable/1556423

ČAPIENĖ, A., \& RAGAUSKAITĖ, A. (2017). Entrepreneurship Education at University: Innovative Models and Current Trends [Educación emprendedora universitaria: modelos innovadores y tendencias actuales]. Research for Rural Development, 2 , 284-291. https://doi.org/10.22616/rrd.23.2017.080

CASTILLO, A. (1999). Estado del arte en la enseñanza del emprendimiento. En: Emprendedores como creadores de riqueza y desarrollo regional, 4-21. http://recursos.ccb.org.co/bogotaemprende/portalninos/contenido/ doczestadodelarteenlaensenanzadelemprendimiento.pdf

CERDA, J., VERA, C., \& RADA, G. (2013). Odds ratio: aspectos teóricos y prácticos. Revista Médica de Chile, 141(10), 1329-1335. https://doi.org/10.4067/So03498872013001000014

CHAPA, E. A. (2008). Programa emprendedor obligatorio. Alternativa de educación integral. Formación universitaria, 1(6), 353-357. https://doi.org/10.4067/S071850062008000600006 
CHIA, C., \& LIANG, C. (2016). Influence of Creativity and Social Capital on the Entrepreneurial Intention of Tourism Students IInfluencia de la creatividad y el capital social en la intención emprendedora de los estudiantes de turismo]. Journal of Entrepreneurship, Management and Innovation (JEMI), 12(2), 151-168. https://doi.org/10.7341/20161227

DENCKER, J. C., GRUBER, M., \& SHAH, S. K. (2009). Pre-entry Knowledge, Learning, and the Survival of New Firms IConocimiento previo al ingreso, aprendizaje y supervivencia de nuevas empresas]. Organization Science, 20(3), 516-537. https://doi.org/10.1287/orsc.1080.0387

DÍAZ-GARCÍA, C., SÁEZ-MARTÍNEZ, F., \& JIMÉNEZ-MORENO, J. (2015). Evaluación del impacto del programa educativo "Emprendedores" en la intención emprendedora de los participantes. RUSC. Universities and Knowledge Society Journal, 12(3), 17-31. https://www.redalyc.org/pdf/780/78038521002.pdf

DOĞAN, E. (2015). The Effect of Entrepreneurship Education on Entrepreneurial Intentions of University Students in Turkey IEl efecto de la educación emprendedora en las intenciones empresariales de los estudiantes universitarios en Turquia]. Ekoist: Journal of Econometrics and Statistics, 23(1), 79-93. https:// dergipark.org.tr/tr/download/article-file/270093

ECHEVERRI-SÁNCHEZ, L., VALENCIA-ARIAS, A., BENJUMEA-ARIAS, M., \& BARRERA-DEL TORO, A. (2018). Factors That Affect the Entrepreneurial Intention in University Students: A Qualitative Analysis [Factores que inciden en la intención emprendedora del estudiantado universitario : Un análisis cualitativo]. Revista electrónica Educare, 22(2), 1-19. https://doi.org/10.15359/ree.22-2.10

FAYOLLE, A., LIÑÁN, F., \& MORIANO, J. A. (2014). Beyond Entrepreneurial Intentions: Values and Motivations in Entrepreneurship IMás allá de las intenciones emprendedoras: valores y motivaciones en el emprendimiento]. International Entrepreneurship and Management Journal, 10(4), 679-689. https://doi. org/10.1007/s11365-014-0306-7

GARCIAA-CABRERA, A. M., DÉNIZ-DÉNIZ, M. C., \& CUÉLLAR-MOLINA, D. (2015). Inteligencia emocional y emprendimiento : posibles lineas de trabajo. Cuadernos de Administración, 28(51), 65-101. https://doi.org/10.11144/Javeriana.cao28-51. ieep

GÓMEZ-ARAUJO, E., LAFUENTE, E., VAILLANT, Y., \& GÓMEZ, L. (2015). El impacto diferenciado de la autoconfianza, los modelos de referencia y el miedo al fracaso sobre los jóvenes emprendedores. INNOVAR. Revista de Ciencias Administrativas y Sociales, 25(57), 157-173. https://revistas.unal.edu.co/index. php/innovar/article/view/51555/51462

GUTIÉRREZ, J., ASPRILLA, E., \& GUTIÉRREZ, J. (2016). Estado del arte del emprendimiento empresarial en materia de ciencia, tecnología e innovación. Revista Universitaria Ruta, 18(2), 1-25. https://revistas.userena.cl/index.php/ ruta/article/view/795

HAIR, J. F., ANDERSON, R. E., TATHAM, R. L., \& BLACK, W. C. (1999). Análisis Multivariante (Quinta). Prentice Hall.

HATTAB, H. W. (2014). Impact of Entrepreneurship Education on Entrepreneurial Intentions of University Students in Egypt IImpacto de la educación emprendedora en las intenciones empresariales de los estudiantes universitarios en Egipto]. The Journal of Entrepreneurship Education, 23(1), 1-18. https://doi.org/10.1177/0971355713513346

HERRERA, R., \& VILLALOBOS, M. (2018). Caracterización de la formación académica en emprendimiento de pregrado que se realiza en universidades chilenas. Formación universitaria, 11(4), 43-52. https://doi.org/10.4067/S071850062018000400043 
HISRICH, R., PETERS, M., \& SHEPHERD, D. A. (2017). Entrepreneurship [Emprendimiento]. McGraw-Hill Education.

HOGARTH, R. M., \& KARELAIA, N. (2012). Entrepreneurial Success and Failure: Confidence and Fallible Judgment [Éxito y fracaso emprendedor: confianza y juicio falible]. Organization Science, 23(6), 1733-1747. https://doi.org/10.1287/ orsc.1110.0702

ISMAIL, A., SAWANG, S., \& ZOLIN, R. (2018). Entrepreneurship Education Pedagogy: Teacher-student-centred Paradox [Paradoja centrada en el profesor y el alumno]. Education \& Training, 60(2), 168-184. https://doi.org/10.1108/ET-072017-0106

KARIM, M. S. A. (2016). Entrepreneurship Education in an Engineering Curriculum [Educación emprendedora en un curriculum de ingenieria]. Procedia Economics and Finance, 35(October, 2015), 379-387. https://doi.org/10.1016/S22125671(16)00047-2

KRUEGER, N. F., \& BRAZEAL, D. V. (1994). Entrepreneurial Potential and Potential Entrepreneurs [Potencial emprendedor y potenciales emprendedores]. Entrepreneurship Theory and Practice, 91-104. https://cemi.com.au/sites/all/ publications/Krueger\%20and\%20Brazeal\%201994.pdf

LAW, K. M. Y., \& BREZNIK, K. (2017). Impacts of Innovativeness and Attitude on Entrepreneurial Intention: Among Engineering and Non-engineering Students Ilmpactos de la innovación y la actitud en la intención emprendedora: entre estudiantes de ingeniería y no ingenieros]. International Journal of Technology and Design Education, 27(4), 683-700. https://doi.org/10.1007/s10798-0169373-0

LIÑÁN, F. (2004). Educación empresarialy modelo de intenciones. Formación para un empresariado de calidad. Análisis empirico para la provincia de Sevilla [Universidad de Sevilla]. https://idus.us.es/xmlui/bitstream/handle/11441/15036/M_TD0503.pdf? sequence $=1$ \&isAllowed $=y$

LIÑÁN, F., \& FAYOLLE, A. (2015). A Systematic Literature Review on Entrepreneurial Intentions: Citation, Thematic Analyses, and Research Agenda IUna revisión sistemática de la literatura sobre intenciones emprendedoras: citas, análisis temáticos y agenda de investigación]. International Entrepreneurship and Management Journal, 11(4), 907-933. https://doi.org/10.1007/s11365-015-03565

LIÑÁN, F., RODRIGUEZ-COHARD, J. C., \& RUEDA-CANTUCHE, J. M. (2011). Factors Affecting Entrepreneurial Intention Levels: A Role for Education [Factores que afectan los niveles de intención emprendedora: un papel para la educación]. International Entrepreneurship and Management Journal, 7(2), 195-218. https:// doi.org/10.1007/s11365-010-0154-Z

MAHENDRA, A. M., DJATMIKA, E. T., \& HERMAWAN, A. (2017). The Effect of Entrepreneurship Education on Entrepreneurial Intention Mediated by Motivation and Attitude among Management Students, State University of Malang, Indonesia [El efecto de la educación emprendedora en la intención emprendedora mediada por la motivación y la actitud entre estudiantes de gestión, Universidad Estatal de Malang, Indonesial. International Education Studies, 10(9), 61. https://doi.org/10.5539/ies.v10ngp61

MAJID, I. A., ISMAIL, K. B., \& COOPER, S. (2011). Prevalence of Entrepreneurial Management Practices in Technology-based Firms in Malaysia [El impacto de la educación emprendedora en la intención emprendedora de los estudiantes de ciencias e ingenieria versus programas universitarios de estudios empresariales]. African Journal of Business Management, 5(6), 2075-2082. https:// academicjournals.org/journal/AJBM/article-full-text-pdf/974CAgD22157 
MARESCH, D., HARMS, R., KAILER, N., \& WIMMER-WURM, B. (2016). The Impact of Entrepreneurship Education on the Entrepreneurial Intention of Students in Science and Engineering versus Business Studies University Programs [El impacto de la educación emprendedora en la intención emprendedora de los estudiantes de ciencias e ingeniería versus programas universitarios de estudios empresariales]. Technological Forecasting and Social Change, 104, 172179. https://doi.org/10.1016/j.techfore.2015.11.006

MCCLELLAND, D. (1961). The Achieving Society [La sociedad del logro]. D. Van Nostrand.

MCGEE, J. E., PETERSON, M., MUELLER, S. L., \& SEQUEIRA, J. M. (2009). EntrepreneurialSelf-efficacy: Refining the Measure [Autoeficacia emprendedora: Refinando la medidal. Entrepreneurship: Theory and Practice, 33(4), 965-988. https://doi.org/10.1111/j.1540-6520.2009.00304.x

MINERA, L. E. (2009). El papel de la motivación y las actitudes en el aprendizaje de ELE en un contexto de enseñanza formal para adultos alemanes. Revista Nebrija de Lingüistica Aplicada, 6, 58-74. http://www.nebrija.com/revista-linguistica/ files/articulosPDF/articulo_53217299c010d.pdf

MORTAN, R. A., RIPOLL, P., CARVALHO, C., \& BERNAL, M. C. (2014). Effects of Emotional Intelligence on Entrepreneurial Intention and Self-efficacy [Efectos de la inteligencia emocional sobre la intención emprendedora y la autoeficacial. Journal of Work and Organizational Psychology, 30(2014), 97-104. https://doi. org/10.1016/j.rpto.2014.11.004

PRASETIO, E. (2016). Assessing the Impact of Entrepreneurship Education on Entrepreneurial Self-efficacy (ESE) and Entrepreneurial Intention (EI): a Longitudinal Study [Evaluación del impacto de la educación en el espíritu empresarial en la autoeficacia emprendedora (ESE) y la intención emprendedora (IE): un estudio longitudinal]. https://doi.org/10.13140/RG.2.1.1969.9607

ROBINSON, P. B., STIMPSON, D. V., HUEFNER, J. C., \& HUNT, H. K. (1991). An Attitude Approach to the Prediction of Entrepreneurship IUn enfoque de actitud para la predicción del espiritu empresariall. Entrepreneurship Theory and Practice, 15(4), 13-31. https://doi.org/10.1177/104225879101500405

SÁNCHEZ, J., WARD, A., HERNÁNDEZ, B., \& FLOREZ, J. (2017). Educación emprendedora: estado del arte. Propósitos y Representaciones, 5(2), 401-473. https://doi.org/10.20511/pyr2017.v5n2.190

SONDARI, M. C. (2014). Is Entrepreneurship Education Really Needed?: Examining the Antecedent of Entrepreneurial Career Intention [¿Se necesita realmente la educación emprendedora?: examinando el antecedente de la intención de carrera emprendedoral. Procedia - Social and Behavioral Sciences, 115(2014), 44-53. https://doi.org/10.1016/j.sbspro.2014.02.414

SORIA-BARRETO, K., ZUÑIGA-JARA, S., \& RUIZ-CAMPO, S. (2016). Educación e intención emprendedora en estudiantes universitarios: un caso de estudio. Formación Universitaria, 9(1), 25-34. https://doi.org/10.4067/S071850062016000100004

SORIANO, D. R., \& CASTROGIOVANNI, G. J. (2012). The Impact of Education, Experience and Inner Circle Advisors on SME Performance: insights from a study of public development centers IEl impacto de la educación, la experiencia y los asesores del círculo intimo en el desempeño de las PYME: ideas de un estudio de centros públicos de desarrollo]. Small Business Economics, 38(3), 333-349. https://doi.org/10.1007/s11187-010-9278-3

VAN PRAAG, C. M., \& CRAMER, J. S. (2001). The Roots of Entrepreneurship and Labour Demand: Individual Ability and Low Risk Aversion [Las raices del emprendimiento y la demanda laboral: capacidad individual y aversión al bajo riesgol. Economica, 68(269), 45-62. https://doi.org/10.1111/1468-0335.00232 
VECIANA, J. M. (1999). Entrepreneurship as a Scientific Research Programme [El emprendimiento como programa de investigación científica]. Revista Europea de Dirección y Economía de la Empresa, 8(3), 23-71. https://doi.org/10.1007/9783-540-48543-8_2

WANG, J. H., CHANG, C. C., YAO, S. N., \& LIANG, C. (2016). The Contribution of Self-efficacy to the Relationship between Personality Traits and Entrepreneurial Intention [La contribución de la autoeficacia a la relación entre rasgos de personalidad e intención emprendedora]. Higher Education, 72(2), 209-224. https://doi.org/10.1007/s10734-015-9946-y

ZHU, F., FAN, S. X., \& ZHAO, L. (2019). Having Entrepreneurial Friends and Following Them? The Role of Friends' Displayed Emotions in Students' Career Choice Intentions liTener amigos emprendedores y seguirlos? El rol de las emociones mostradas por los amigos en las intenciones de elección de carrera de los estudiantes]. Journal of Enterprising Culture, 27(4), 445-470. https://doi. org/10.1142/s021849581950016x 